\title{
Head Elevation by Ten Degrees in Prone Position Surgery Consistently Decreases and Minimizes Variation in Intraocular Pressure (IOP)
}

\author{
Matthew B. Ellison, $M D^{1 *}$, Brian W. Grose, $M D^{1}$, Pavithra Ranganathan, $M D^{1}$, Manuel C Vallejo, $M D$, \\ $D M D^{1}$, Ralph Harding, $D O^{3}$, Hong Wang, $M D^{1}$ and Sanford E. Emery, MD, MBA
}

${ }^{1}$ Department of Anesthesiology, West Virginia University, Morgantown, WV, USA

${ }^{2}$ Department of Orthopaedics, West Virginia University, Morgantown, WV, USA

${ }^{3}$ Department of Surgery, Carl Vinson VA Medical Center, Dublin, GA, USA

\begin{abstract}
Purpose: The aim of this study is to explore the effect of 10 Degree head elevation in prone position procedures on Intra-ocular pressure (IOP).

Methods: A prospective, randomized observational study in 52 patients undergoing lumbar spine surgery in a tertiary care academic center, West Virginia University Hospital Patients $(n=52)$ between the ages of 18 and 80 undergoing lumbar spine fusion procedures in the prone position under standardized general anesthetics. Exclusion criteria were a diagnosis of tumor, infection, traumatic injury, a history of eye disease, ocular surgery, cervical spine surgery, chronic neck pain, or cervical stenosis. Patients were randomized into one of two treatment groups for surgery:
\end{abstract}

Group 1: Head neutral prone position

Group 2: 10 Degree head elevation in prone position

Results: IOP was measured at specific, defined intervals including: prior to induction, supine after induction, after prone positioning and at defined intervals throughout the procedure, and after supine positioning at conclusion of the surgery. Corresponding blood pressure and $\mathrm{pCO}_{2}$ measurements were recorded at each time point.

Conclusions:

- The 10-degree head elevation position significantly decreased the rate of increase of IOP in as early as 30 minutes in the prone position.

- A lower degree of variation and increased consistency in IOP was found in patients in the 10-degree head elevation group compared to the control group.

\section{Keywords}

Intraocular pressure (IOP), Perioperative visual loss (POVL), Ischemic optic neuropathy (ION), Prone position

\section{Introduction}

Visual loss is a rare but disastrous complication that has been associated with several specific types of surgery and has been ranked as a major patient-safety issue among anesthesiologists [1]. Although difficult to quantify, the incidence of perioperative visual loss (POVL) is estimated to range from $0.0003 \%-1 \%$, depending on the type of surgery [2-4]. It has been reported in patients undergoing cardiac, general, and orthopedic surgery with the highest incidence occurring in cardiac $(8.64 / 10,000)$ and spinal fusion $(3.09 / 10,000)$ surgeries [2]. Based on the incidence alone, it is not surprising that a great number of POVL cases in the literature are related to spine and cardiac surgery [57]. Provider and public appreciation of vision loss as a possible risk of surgery has only occurred in the last few decades, with the first case of visual loss following spine surgery reported in the 1950's by Hollen horst, et al. [8]. Although the most common ophthalmologic cause of perioperative visual loss is ischemic optic neuropathy (ION), it may also be caused by cortical blindness, central retinal artery occlusion, and external injuries $[9,10]$. ION can be classified as either anterior or posterior ischemic optic neuropathy (PION). The anterior variant typically affects the optic disc or the junctional area next to it. PION affects the mid-orbital region of the optic nerve between the optic foramen and the central retinal artery's entry [10].

Previously published data from our group (Emory, et al.) examining the effect of head position on intraocular pressure (IOP) during lumbar spine fusion in the prone position showed that head elevation decreased IOP compared to the neutral position [11]. Similarly, other studies have demonstrated that IOP increases after prone positioning in both awake and anesthetized subjects $[11,12]$. This increase in IOP may lead to decreased perfusion to the optic nerve and subsequently, ischemic optic neuropathy [13].

The aim of this study is to explore and analyze data collected by our study group (Emory, et al.) to see if there were findings of clinical significance to improve care and 
outcomes in prone procedures and to hypotheses for further research.

\section{Methods}

This prospective trial was approved by our Institutional Review Board and registered at Clinicaltrials.gov (\#NCT02342288). Patients were randomized into one of two groups.

Group 1: Patients in the head neutral prone position

Group 2: Patients in the 10-degree head elevation prone position

Each patient in the study received standardized care with their head in the neutral or 10-degree head elevated position in a prone face down position during surgery. The study population included male and female patients between the ages of 18 and 80 undergoing lumbar spine fusion procedures. Both primary and revision surgical cases were included. All patients underwent instrumented fusion with allograft or autograft bone, with or without transforaminal lumbar interbody fusion (TLIF) constructs. Patients undergoing thoracolumbar procedures or anterior-posterior $\left(360^{\circ}\right.$-degrees) procedures were excluded. In addition, patients with a diagnosis of tumor, infection, traumatic injury, history of eye disease, history of ocular surgery, prior cervical spine surgery or history of chronic neck pain or cervical stenosis were also excluded. Twenty-seven qualified patients were consented and enrolled in the study by a research coordinator during their orthopedic clinic visits in Group 1 and twenty five in group 2.

Group 1: All of the patients' heads were maintained in the neutral position throughout surgery utilizing Gardner-Wells tong traction with ten pounds of weight. Neck flexion and extension were minimized such that the head was in line with the thorax. Additionally, the operating table was kept level in all cases.

Group 2: All the patients' heads were maintained in the 10-degree elevated position (as measured by a large goniometer) throughout surgery utilizing Gardner-Wells tong traction with ten pounds of weight. Neck flexion and extension were minimized such that the head was maintained in-line with the thorax. Additionally, the operating table was kept at a 10-degree incline in all cases.

To provide consistency, all procedures were performed by three orthopedic spine surgeons and all anesthetics were performed by a small group of four anesthesiologists. One research technician obtained all IOP measurements with an applanation tonometer (Tono-Pen, Reichert Technologies, Depew, NY). This is a standard device for measuring IOP that involves gently touching the tonometer to the cornea.

Measurements on both eyes were obtained at the following time $(T)$ points for all patients in the study: T1) sitting in hospital bed in the pre-operative holding area, T2) following induction of anesthesia in the operating room in the supine position, T3) five minutes after prone positioning on the Jackson table, T4) measurements taken every 15 minutes during the case while in the prone position. Once three values in a row were within three $\mathrm{mmHg}$ of each other, the technician began measurements again after one hour. After this hour hiatus, readings were again taken every 15 minutes, and if three consecutive readings were within three $\mathrm{mmHg}$, another one-hour hiatus was taken. Measurements continued in this manner until the completion of each case. T5) A final post-operative reading was taken after five minutes of equilibration in the supine position while the patient was still anesthetized. No further readings were obtained after surgery. For the initial IOP measurement in the holding area, both eyes were topically anesthetized with $0.5 \%$ tetracaine hydrochloride drops, and baseline IOP was measured in the sitting position before premedication. The eyes were taped shut during surgery with lifting of the tape to conduct the tonometer measurements. Taping allows the eye to remain lubricated naturally with tears during the surgery. Four measurements per eye were taken at each time interval in the study. When the IOP measurements were conducted, corresponding vital signs including blood pressure, heart rate, pulseoximetry, temperature and $\mathrm{CO}_{2}$ values were recorded. Additional data points including duration of surgery, colloid and crystalloid amounts, estimated blood loss, transfusions, and gender were recorded for each case and analyzed for their effect on IOP. In the recovery room, patients were asked about any vision changes or eye discomfort.

The anesthetic technique was standardized for each procedure. For all patients, pre-operative hemoglobin and hematocrit laboratory values were obtained. Preoperative medications were limited to midazolam $0.02 \mathrm{mg} / \mathrm{kg}$.

\section{Anesthetic protocol}

A standardized intravenous induction technique was utilized consisting of lidocaine $1 \mathrm{mg} / \mathrm{kg}$, propofol $2 \mathrm{mg} / \mathrm{kg}$, fentanyl $1 \mathrm{mcg} / \mathrm{kg}$, and rocuronium $0.6 \mathrm{mg} /$ $\mathrm{kg}$. During the surgical procedure, rocuronium (0.1-0.2 $\mathrm{mg} / \mathrm{kg}$ ) was re-dosed to maintain a minimum of 1 out of 4 twitches on the peripheral nerve stimulator. After endotracheal intubation but prior to prone positioning, an arterial line was placed in either the right or left radial artery. Study parameters for intraoperative maintenance included: crystalloid infusion of less than or equal to three liters, ventilator tidal volume at six to ten $\mathrm{cc} / \mathrm{kg}$, peak airway pressures between 25 and $35 \mathrm{mmHg}$, five $\mathrm{mmHg}$ positive end expiratory pressure 
(PEEP), end tidal carbon dioxide $\left(\mathrm{ETCO}_{2}\right)$ between 30 and $34 \mathrm{mmHg}$, and Sevoflurane concentration between 0.8 to $1.3 \mathrm{MAC}$. Blood pressure was maintained within $\pm 25 \%$ of the patient's preoperative reading along with a MAP above $60 \mathrm{mmHg}$. If required, this was accomplished with a phenylephrine infusion that was initiated at $0.3 \mathrm{mcg} / \mathrm{kg} / \mathrm{min}$ and titrated to maintain the listed values. Intraoperative hemoglobin was measured at a minimum of every two hours utilizing arterial blood gas analysis. If hemoglobin was determined to be less than $10 \mathrm{~g} / \mathrm{dl}$, packed red blood cells were given and the value was rechecked. A Bispectral (BIS) monitor was placed on each patient prior to prone positioning and the levels were maintained between 40-60 during the case. At the end of the procedure, reversal of the neuromuscular block was accomplished utilizing intravenous neostigmine $0.06 \mathrm{mg} / \mathrm{kg}$ and glycopyrrolate $0.01 \mathrm{mg} / \mathrm{kg}$. Post-operative pain control was managed with the use of intravenous hydromorphone PCA set to deliver $0.2 \mathrm{mg}$ every 6 minutes with a $2 \mathrm{mg}$ lockout.

\section{Statistics}

\section{Sample size calculation}

A power analysis performed prior to initiation of the study demonstrated that, if the change in the intervention group was 1.5 times the standard deviation larger than the change in the control group, then the proposed sample size of fifty-two subjects would result in $89 \%$ power for the study.

The relationship between variance of the slope for the neutral and inclined groups was explored. We defined a rudimentary slope $m$ for patient $i$ as

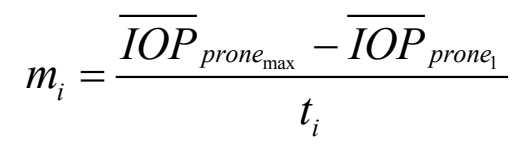

Where $\overline{I O P}_{\text {prone }_{\max }}$ is the maximum mean of the right and left eye IOP observed while the patient is prone, $\overline{I O P}_{\text {prone }_{1}}$ is the first mean IOP observation made while prone, and $t_{i}$ is the number of minutes patient $i$ was in the prone position. This calculation leaves out the IOPs observed during supine position.

After testing for normality, the $F$-test was used to compare the variance of the slopes for the Neutral group to that of the Inclined group. Loess smoothing was performed for all observations of patients in each group starting at the first prone observation there was a gradual rise in IOP over time. The data was analyzed using analysis of covariance (ANCOVA) in order to compare the two groups while controlling for covariate(s). The single out come measure determining whether the result of the trial would be positive or negative was the change in the two-eye average IOP; that is, the difference between the baseline two-eye average, measured with the patient prone, and the maximum two-eye average measured in the observation period. Descriptive statistical analyses included the mean, standard deviation, and range for the IOPs measured at each of the time points in the control and intervention groups. Independent variables included age, duration of the operation, blood loss, type and amount of fluid replacement, systolic blood pressure, diastolic blood pressure, $\mathrm{PCO}_{2}$, sex, and head position (neutral or elevated). Inferential statistical analysis included independent $t$ tests, regression analysis, and ANCOVA. The mean and standard deviation for the total time of procedure and time in prone position was tabulated and the $p$-value for the difference of means between the groups was calculated using Student's $t$-test.

\section{Results}

Fifty-two patients were randomly assigned to one of two treatment groups, Neutral $\left(n_{N}=27\right)$ or Incline $\left(n_{1}=\right.$ $25)$ during surgery. The initial average of the two eye IOP readings in the pre-operative holding area in the sitting position was $16.93 \pm 3.33 \mathrm{mmHg}$ for both groups and no significant difference was found in both groups. After induction of anesthesia in the supine position while in the OR, the average IOP value was $14.24 \pm 4.96$ $\mathrm{mmHg}$ and no significant difference was found between the two groups. The first IOP measurement obtained in the prone position was $23.96 \pm 4.93 \mathrm{mmHg}$. The mean value for the change in IOP measurements (i.e. both eyes average maximum IOP - both eyes average baseline IOP obtained at first prone measurement) was $12.7 \pm 7.85 \mathrm{mmHg}$ for neutral and $6.74 \pm 5.48 \mathrm{mmHg}$ for 10-degree elevation.

After testing for normality, the $F$-test was used to compare the variance of the slopes for the Neutral group to that of the Inclined group. With $F(26,24)=$ 2.35 and $P=0.0385$, the evidence supports the claim that the variance in rate of change in IOP based on this definition is lower for the elevated group.

As seen in Figure 1, (Loess smoothing was performed for all observations of patients in each group starting at the first prone observation there was a gradual rise in IOP over time). The change in IOP, compared to the baseline prone measurement, became statistically significant approximately 30 minutes into the cases.

The average of all prone IOP values for the entire case for neutral prone was: $31.6 \pm 6.79 \mathrm{mmHg}$ and 10-degree elevation were: $25.04 \pm 4.83 \mathrm{mmHg}$. For any point in time after 30 minutes, this statistically significant difference was maintained throughout the operative procedure (Table 1 and Table 2). Overall, the $I O P$, the rate of increase, and the variation in the value of IOP was significantly less in the group with 10-degree head elevations (Figure 1 and Figure 2). There was no difference in the mean times for the 2 groups. 


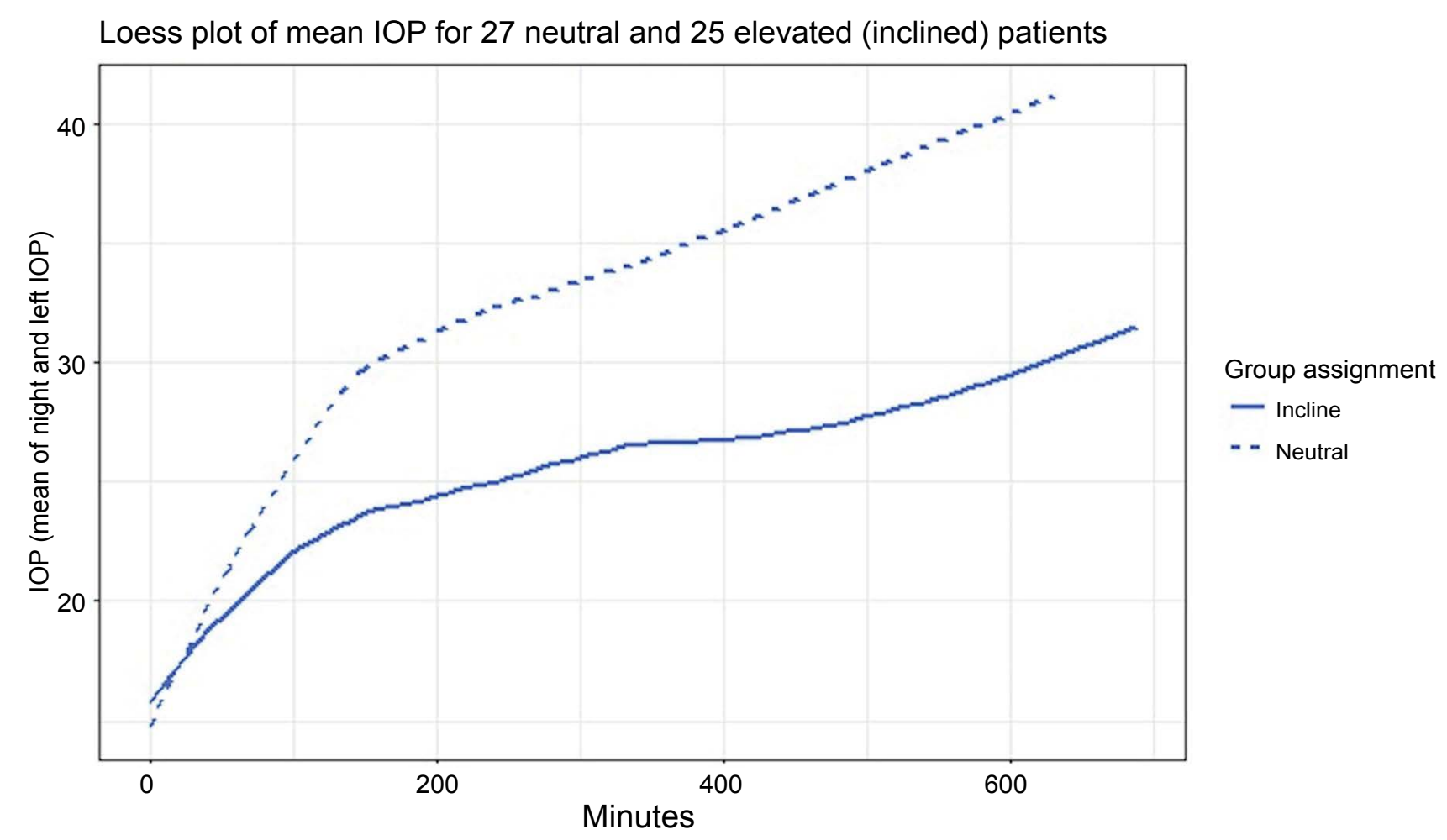

Figure 1: Loess plot of mean IOP for 27 neutral and 25 elevated (inclined) patients. The mean IOPs for both eyes are plotted at each time interval in both the neutral and head elevated positions.

\section{Change in IOP over time}

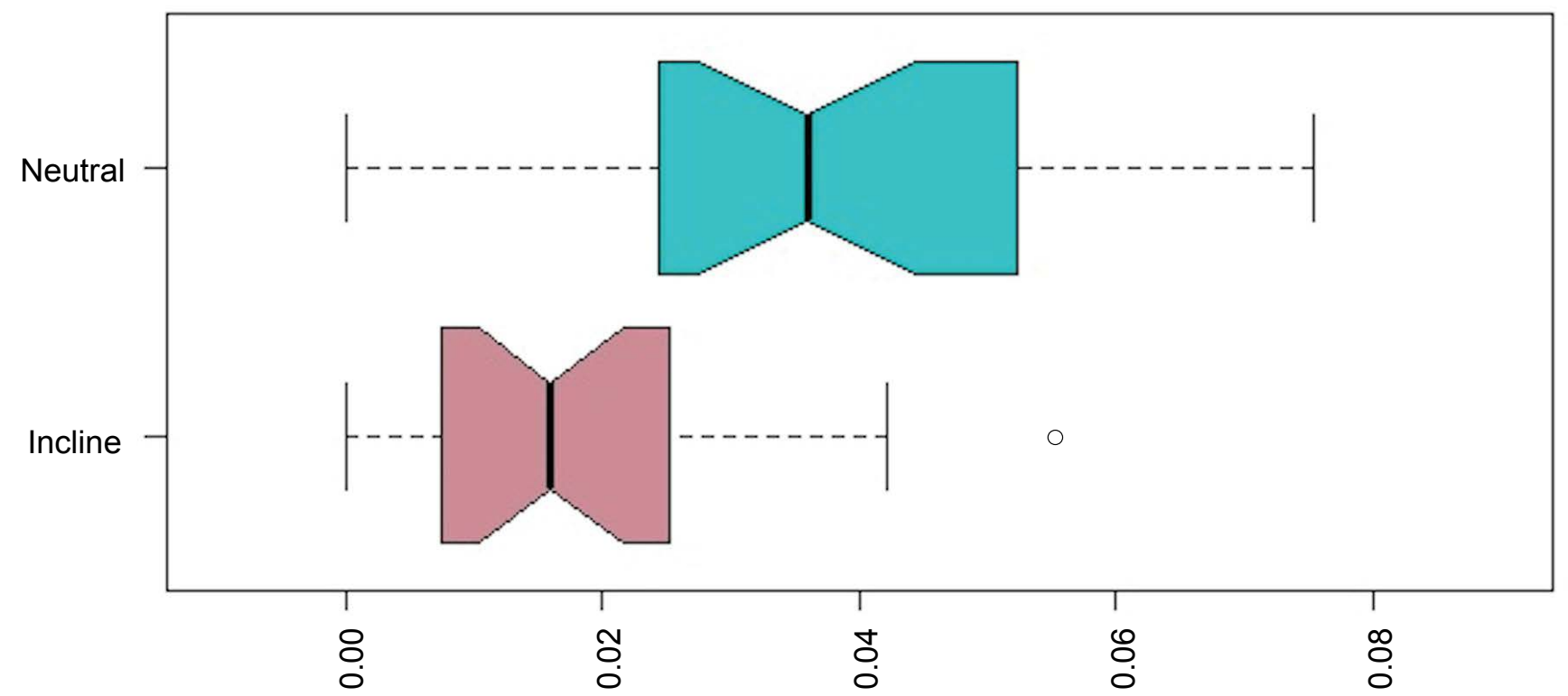

Figure 2: Change in IOP over time. This figure demonstrates that the IOP changes to a greater degree in the neutral position and remains relatively stable in patients in the prone position.

Table 1: Data summary for procedure times for each subject in both head neutral and head elevated positions.

\begin{tabular}{|l|l|l|l|l|l|}
\hline & \multicolumn{3}{|c|}{ Neutral $(\mathbf{n}=\mathbf{2 7})$} & \multicolumn{3}{c|}{ Elevated (n= 25) } & t-test & p-value \\
\hline & Mean & SD & Mean & SD. & 0.2461 \\
\hline Total time of procedure & 347.74 & 97.68 & 379.72 & 98.60 & 0.6267 \\
\hline Total time prone & 227.30 & 97.50 & 240.52 & 97.22 & 0.62 \\
\hline
\end{tabular}


Table 2: Data Summary of Intraocular pressures. Prone $1^{\text {st }}$ IOP is the $1^{\text {st }}$ intraocular pressure measured after the patient is placed in prone position. The prone all pressure is the average of all intraocular pressures throughout the case in both the neutral and head elevated positions.

\begin{tabular}{|c|c|c|c|c|c|}
\hline & Neutral & & Elev. 10 degrees & & $p$-value \\
\hline & Mean $(\mathrm{mmHg})$ & Range (+/-) & Mean $(\mathrm{mmHg})$ & Range (+/-) & \\
\hline Prone $1^{\text {st }}$ IOP & 23.6 & 5.4 & 22.4 & 3.8 & 0.3455 \\
\hline Prone All & 31.6 & 6.79 & 25 & 4.83 & $<0.0001$ \\
\hline Mean Difference & 12.7 & 7.85 & 6.74 & 5.48 & 0.0025 \\
\hline$\left(\operatorname{Max}-1^{\text {st }}\right)$ & & & & & \\
\hline
\end{tabular}

The duration of surgery was the only factor that significantly influenced the change in IOP over time. Colloid and crystalloid amounts, estimated blood loss, transfusions, and gender had no correlation with the rise in IOP over time. No patient reported visual changes or visual loss immediately after surgery, prior to hospital discharge, and at follow up a minimum of six months after the operation. One patient sustained a mild corneal abrasion that resolved within 24 hours using ophthalmic ointment.

\section{Discussion}

Our study indicates that a simple measure of head elevation to 10-degree can decrease the rise and rate of rise of IOP in prone position surgery. This is especially valuable for prone position surgery that is longer than 30 minutes.

Perioperative visual loss (POVL) is an infrequent but potentially debilitating complication of surgery with an incidence estimated to range from $0.0003 \%-1 \%$ [24]. According to multiple studies, it appears to occur with greater frequency in spine surgery $(0.2 \%)$ than in cardiac surgery $(0.11 \%)$ or general surgery $(0.0008 \%)$ $[4,14]$. The incidence is also very low in intracranial neurosurgical procedures, which may be explained in a recent study by Czorlich, et al. He demonstrated that the dura opening and subsequent loss of cerebrospinal fluid in intracranial procedures leads to a decrease in IOP [15]. The most frequent cause of visual disturbance after prone spine surgery is corneal abrasion, although it doesn't usually lead to permanent visual field defects or vision loss [10]. Other causes of POVL include: cortical blindness, central retinal artery occlusion, and ischemic optic neuropathy $[2,10]$. Direct pressure on the globe, often due to incorrect positioning of the headrest while in the prone position, may lead to unilateral vision loss due to central retinal artery occlusion and often presents with periorbital edema [10]. Although POVL after spine surgery can result from retinal ischemia or cortical blindness, it is most frequently caused by ION and is often bilateral $[6,9]$. ION is divided into either anterior ischemic option neuropathy (AION) or posterior ischemic optic neuropathy (PION) based on the location of the defect in the optic nerve [10]. In
AION, the defect typically occurs in the optic disc or the adjacent junctional area in a watershed zone of the anterior optic nerve whose blood supply originates from branches of the posterior ciliary artery $[2,13,16]$. When ischemia occurs in the intraorbital portion of the optic nerve, located between the optic foramen and the entry of the central retinal artery, it is characterized as posterior ischemic optic neuropathy (PION). This portion of the optic nerve receives its blood supply from terminal centripetal pial vessels $[10,17]$.

The mechanisms of perioperative ION are controversial and likely multifactorial, possibly resulting from decreased perfusion to the optic nerve [18]. Ocular perfusion pressure is the difference between the ciliary artery pressure in the optic nerve and the venous drainage of the eye, which is commonly expressed as mean arterial pressure (MAP) minus IOP $[11,19]$. Increased IOP is a risk factor for ischemic optic neuropathy and a possible mechanism for perioperative visual loss [13]. IOP may become even more important when determining the blood supply to the optic nerve if both the MAP and hematocrit are low. Prone positioning, both awake and under general anesthesia, has been shown to increase IOP, therefore decreasing perfusion pressure to the optic nerve despite maintenance of normotension $[11,13,18]$. This may lead to areas of ischemia in the watershed region around the posterior optic nerve and possibly result in visual loss [18]. Episcleral venous pressure (EVP) may influence IOP by the formula IOP = (rate of aqueous humor production/facility of outflow)/EVP, which is known as the Goldmann equation [13]. Any increase in central venous pressure or shift in venous blood volume, such as occurs in prone or head down positioning, will increase EVP (and subsequently IOP) because the episceral veins are directly connected to the central circulation through a valve-less system. Greater than three-quarters of the aqueous humor outflow travels through Schlemm's canal into the episcleral veins [13]. Even small increases in aqueous humor volume may cause tremendous changes in IOP because the eye is a somewhat restricted, non-distensible space. Head elevation to decrease the variation and to minimize the increase in IOP can be very beneficial by possibly improving aqueous humor outflow and keeping the intra 
ocular volume stable and without wide variations. This could be protective in preventing visual disturbances related to increase in IOP.

The American Society of Anesthesiologists has developed a Task Force on Perioperative Visual Loss which has released several practice advisories with the most recent version coming in 2019. These reports are intended to provide evidence-based recommendations to assist in decision making when caring for patients who may be at risk for POVL [5]. According to the advisory, the performance of a focused preoperative evaluation for POVL has not been studied. However, observational studies have identified preoperative patient risk factors that my increase the risk of POVL [5]. These include preoperative anemia, vascular risk factors including hypertension, diabetes, peripheral vascular disease, coronary artery disease, previous stroke, carotid artery stenosis, obesity, tobacco use, male sex, and diabetic retinopathy $[6,18]$. When positioning the patient after induction of anesthesia, it is important to avoid direct pressure on the eye to prevent central retinal artery occlusion [5].

Intraoperative management to decrease the risk of POVL consists of management of intraoperative fluids (avoid administering large volumes of crystalloid), management of anemia, use of vasopressors to maintain mean arterial pressure at or above the patient's baseline, positioning the patient's head at or above the level of rest of the body, avoiding extensive blood loss, and staging of prolonged procedures $[5,10]$. Hemoglobin or hematocrit values should be monitored periodically during surgery in high-risk patients who experience substantial blood loss and transfusion should be considered to optimize the hematocrit in high risk patients $[5,10]$. There are some providers who advocate for routine use of invasive arterial blood pressure monitoring during the case so that hypotension, hypovolemia, and anemia can be corrected more quickly [20]. Intraoperative measurement of IOP in high risk patients could also be considered, as our study and others have successfully demonstrated that this can be done safely. Yoshimura, et al., demonstrated that intraoperative IOP measured at one hour after prone positioning can be an accurate predictor of a future
IOP values greater than $30 \mathrm{mmHg}$. This proved that early measurement of IOP can be used as a predictor of dangerously high levels further in the case [21]. It is recommended that the patient's head be elevated in the recovery area unless supine or trendelenberg position is required secondary to hypotension. Patients who considered to be at high-risk for POVL should have their vision evaluated after emergence from anesthesia by an ophthalmologist [5].

Although there is no proven therapy for POVL, it may be reversible in the early stages if caused by ION. Therefore, vigilance, assessment, and immediate action are necessary when postoperative visual disturbances occur after spine surgery $[5,18]$. An urgent ophthalmic exam should be obtained because emergent correction of anemia, poor hemodynamic status, and arterial oxygenation may improve the prognosis of POVL $[5,22]$. An additional consideration would be to obtain an emergent CT or MRI to rule out intracranial causes of visual loss [5]. Although administration of antiplatelet agents, steroids, or ophthalmic medications to lower IOP have been suggested, there is no evidence that they are beneficial according to the Task Force in the treatment of perioperative ION [5]. Even with these recommendations, the prognosis of PVL is poor, with permanent visual loss in most cases.

Duration of surgery was a factor that significantly influenced the increase in IOP over time in our study. Long duration of surgery has previously been implicated as a risk factor for POVL [2]. The quantity of colloid and crystalloid infused, the estimated blood loss, the presence of blood transfusions, and patient gender had no correlation with the rise in IOP over time in this study. Previous studies have linked the development of POVL to the intraoperative administration of large volumes of crystalloid. However, as previously identified by our group (Emery, et al.) [11], our study protocol limited the crystalloid infused during surgery to three liters followed by colloid/blood administration. This may have led to more judicious approach to fluid management in the operating room. Other limitations of this study include the non-consecutive nature of the study cases, which was primarily due to patient recruitment and the limited availability of the research technician

Table 3: Risk factors for Perioperative Visual Loss (POVL).

\begin{tabular}{|l} 
Preoperative anemia \\
Vascular risks factors including hypertension, diabetes, peripheral vascular disease, coronary artery disease, previous stroke, \\
carotid artery stenosis \\
\hline Obesity \\
\hline Tobacco Use \\
\hline Male sex \\
\hline Diabetic retinopathy \\
\hline Prolonged duration of surgery, often defined as $>5.5$ hours \\
\hline Substantial intraoperative blood loss, often defined as $>800 \mathrm{~mL}$ \\
\hline Intraoperative patient positioning resulting in direct pressure to the eyes \\
\hline
\end{tabular}


Table 4: Strategies for reducing Perioperative Visual Loss (POVL).

Intraoperative head elevation by 10 degrees while in prone position

Correct intraoperative anemia based on age and co-morbidities

Maintain Mean arterial pressure near baseline for adequate cerebral perfusion pressure

High risk individuals should have aggressive correction of hypotension and anemia

Consider intraoperative use of colloids for volume replacement

Consider staging of prolonged surgical procedures in high risk patients

and anesthesiologist. Although previous studies have identified an increase in IOP over time with less than five measurement points, our study tracked the trend in IOP as measured at multiple, defined intervals throughout the duration of surgery. Our study illustrates a steady increase in bilateral IOP after prone positioning at each defined time point throughout the procedure. This supports the theory that prolonged duration of surgery is an independent risk factor for the development of perioperative visual loss, especially in high-risk patients in the prone position (Table 3 and Table 4).

In conclusion, our study indicates that a head elevation to 10-degree can decrease the rise and rate of rise of IOP in prone position surgery with less variability and more stability in IOP which is especially valuable for prone position surgery that is longer than 30 minutes.

\section{References}

1. Stoelting RK. APSF survey results identify safety issues priorities. Anesthesia Patient Safety Foundation Newsletter. 1999; 14:6-7.

2. Shen $Y$, Drum M, Roth $S$. The prevalence of perioperative visual loss in the United States: A 10-year study from 1996 to 2005 of spinal, orthopedic, cardiac, and general surgery. Anes and Analg. 2009; 109(5):135-45.

3. Roth S, Thisted RA, Erickson JP, et al. Eye injuries after non-ocular surgery: a study of 60,965 anesthetics from 1988 to 1992 . Anesthesiology. 1996; 85:1020-7.

4. Warner ME, Warner MA, Garrity JA, et al. The frequency of perioperative vision loss. Anesth Analg. 2001; 93:1417-21.

5. Practice Advisory for Perioperative Visual Loss Associated with Spine Surgery 2019: An Updated Report by the American Society of Anesthesiologists Task Force on Perioperative Visual Loss, the North American NeuroOphthalmology Society, and the Society for Neuroscience in Anesthesiology and Critical Care. Anesthesiology. 2019; 130(1):12-30.

6. Lee LA, Roth S, Posner KL, Cheney FW, Caplan RA, Newman NJ, Domino KB. The American Society of Anesthesiologists Postoperative Visual Loss Registry: analysis of 93 spine surgery cases with postoperative visual loss. Anesthesiology. 2006; 105(4):652-9.

7. Nuttall GA, Garrity JA, Dearani JA, Abel MD, Schroeder DR, Mullany CJ. Risk factors for ischemic optic neuropathy after cardiopulmonary bypass: a matched cases/control study. Anesth Analg 2001; 93:1410-1416.

8. Hollenhorst RW, Svien HJ, Benoit CF. Unilateral Blindness occurring during anesthesia for neurosurgical operations. AMA Arch Opthalmol. 1954; 52:819-30.
9. Myers MA, Hamilton SR, Bogosian AJ, et al. Visual loss as a complication of spine surgery. A review of 37 cases. Spine. 1997; 22:1325-9.

10. Baig MN, Lubow M, Immesoete P, Bergese SD, Hamdy $E A$, Mendel $E$. Vision loss after spine surgery: review of the literature and recommendations. Neurosurg Focus.2007;23(5):1-9.

11. Emery SE, Daffner SD, France JC, Ellison M, Grose BW, Hobbs GR, Clovis NB. Effect of Head Position on Intraocular Pressure During Lumbar Spine Fusion: A Randomized, Prospective Study. J Bone Joint Surg Am. 2015 Nov 18;97(22):1817-23.

12. Pan J, Cheng D, Feng $X$, et al. Effect of body position on intraocular pressure in silicone oil tamponade eyes. Retina. 2018 May;38(5):939-944.

13. Cheng MA, Todorov A, Tempelhoff R, McHugh $T$, Crowder CM, Lauryssen $\mathrm{C}$. The effect of prone positioning on intraocular pressure in anesthetized patients. Anesthesiology. 2001;95(6);1351-5.

14. Ozcan MS, Praetel C, Bhatti MT, Gravenstein N, Mahla $\mathrm{ME}$, Seubert $\mathrm{CN}$. The effect of body inclination during prone positioning on intraocular pressure in awake volunteers: a comparison of two operating tables. AnesthAnalg. 2004;99(4):1152-8.

15. Czorlich P, Kratzig T, Kluge N, et al. Intraocular pressure during neurosurgical procedures in context of head position and loss of cerebrospinal fluid. J Neurosurg. 2018 Aug 24:1-10.

16. Newman NJ. Perioperative visual loss after nonocular surgeries. Am J Ophthalmol 2008;145:604.

17. Hayreh SS. Anterior ischemic optic neuropathy. Clinical Neuroscience 1997;4:251-63.

18. Williams EL, HartWMJr, Tempelhoff R. Postoperative ischemic optic neuropathy. Anesth Analg 1995;80:101829.

19. Patil CG, Lad EM, Lad SP, Ho C, Boakye M. Visual loss after spine surgery: a population-based study. Spine. 2008;33(13):1491-6.

20. Epstein NE. How to avoid perioperative visual loss following prone spinal surgery. Surg Neurol Int. 2016 May 17;7(Suppl 13):S328-30.

21. Yoshimura K, Hayashi $H$, Tanaka $Y$, et al. Evaluation of predictive factors associated with increased intraocular pressure during prone position spine surgery. J Anesth. 2015 Apr;29(2):170-4.

22. Hayreh SS. Blood flow in the optic nerve head and factors that may influence it. Prog Retin Eye Res. 2001;20(5):595624. 
Corresponding Author: Matthew B. Ellison, $M D$, Department of Anesthesiology, West Virginia University, PO Box 8255, 1 Medical Center Drive, Morgantown, WV, 26506, USA, Tel: 304-285-1931, Fax: 304-598-4930, E-mail: mellison@wvumedicine.org

Editor: Renyu Liu, MD, PhD, Associate Professor, Department of Anesthesiology and Critical Care, Perelman School of Medicine at the University of Pennsylvania, 336 John Morgan building, 3620 Hamilton Walk, Philadelphia, PA 19104, USA, E-mail: liur@uphs.upenn.edu

Additional publication details

Journal short name: Transl Perioper \& Pain Med

Received Date: December 13, 2018

Accepted Date: February 07, 2019

Published Date: February 22, 2019
Citation: Ellison MB, Grose BW, Ranganathan P, Vallejo MC, Harding R, et al. Head Elevation by Ten Degrees in Prone Position Surgery Consistently Decreases and Minimizes Variation in Intraocular Pressure (IOP). TransI Perioper \& Pain Med 2019; 6 (2):39-46

Copyright: (C) 2019 Ellison MB, et al. This is an open-access article distributed under the terms of the Creative Commons Attribution License, which permits unrestricted use, distribution, and reproduction in any medium, provided the original author and source are credited. 